presently envisaged. I am worried that the plume that generated the Walvis Ridge in the South Atlantic does not appear to have been anywhere near the centre of what I take to be the topographic uplift in the adjacent continents. The Transantarctic Mountains have so far not been included in the model at all, and their exclusion from the Karoo plume on the grounds of geochemical difference is hardly fair, because many of the geochemical characters of continental flood basalts are not related to the plume itself but are generated by interaction with lithospheric rocks. These are not important criticisms, merely indications of some of the large number of exciting problems that can now be attacked within the unifying framework which has been presented.

K.G. Cox is in the Department of Earth Sciences, University of Oxford, Parks Road, Oxford OX1 3PR, UK.

1. White, R.S. \& McKenzie, D.P. J. geophys. Res. 94, 76857730 (1989).

2. White, R.S. \& McKenzie, D.P. Scient. American 261 62-67 (1989)

3. McKenzie, D.P. \& Bickle, M.J. J. Petrol. 29, 625-679 (1988)

4. White, R.S. Nature 327, 191 (1987)

\title{
Export-import family expands
}

\section{Chris Higgins}

WHAT is the connection between such topical subjects as yeast mating type, resistance of human tumours to chemotherapy, protein secretion, bacterial transport and the failure of chloroquine as an antimalarial drug? Whereas this question sounds obscure enough to be borrowed from Round Britain Quiz, the answer is readily apparent from two new papers $^{1,2}$ including that by McGrath and Varshavsky on page 400 of this issue ${ }^{2}$. Each process involves a member of a family of closely related proteins, all of which are believed to be associated with membranes and to bind ATP. These proteins have now been identified in bacteria, yeasts, protozoans, insects and mammals and their similarities imply that each functions by a rather similar mechanism.

Perhaps the most familiar member of this family is the P-glycoprotein or MDR (multidrug resistance) protein. Overexpression of MDR protein in mammalian tumours is responsible for the simultaneous development of resistance to a variety of apparently unrelated chemotherapeutic drugs. The MDR protein consists of two hydrophobic domains, each of which includes about six potential membranespanning helices, and two hydrophilic ATP-binding domains ${ }^{3-5}$. All available evidence is consistent with the view that MDR protein pumps drugs out of cells, reducing their intracellular concentration and, hence, their toxicity. The intriguing similarity in sequence and domain organization between MDR protein and a group of well-characterized, ATP-dependent proteins that are known to be involved in the transport of small molecules across bacterial cell membranes implies that MDR functions by a similar mechanism ${ }^{6-8}$.

Three differences do, however, exist: the domains of the bacterial transport systems are present as separate polypeptides rather than a single multifunctional polypeptide like MDR protein; the bacterial systems transport substrate into the cell while MDR protein pumps substrate out- wards; and the bacterial systems require an additional protein (the periplasmic binding protein) to deliver substrates to the membrane-associated proteins. These differences probably reflect diversification of a single common transport mechanism.

Just as tumour chemotherapy is hindered by multidrug resistance, so the control of malaria has been impeded by the development of chloroquine resistance (CQR) in Plasmodium falciparum. CQR shares many of the characteristics of MDR found in multidrug resistance in tumours and Foote $e t a l .{ }^{1}$ have recently isolated a gene from $P$. falciparum that encodes a homologue of the MDR protein. Not only does the protein encoded by the $P$. falciparum gene have a very similar sequence and domain structure to MDR protein but its gene is amplified in several chloroquineresistant cell lines, just as $m d r$ is amplified in multidrug-resistant tumour cell lines. It seems reasonable to assume that the $C Q R$ protein pumps chloroquine out of parasite cells much as MDR protein pumps drugs out of tumour cells. Although, of course, there are many transport systems in both eukaryotes and prokaryotes that rely on quite different proteins, it seems that the MDR and CQR proteins and a group of bacterial transporters comprise a single family of transport proteins. Other members of this family may include the white and brown loci of Drosophila, which are required for the laying down of eye pigments and are related by sequence to $m d r$.

Many questions remain unanswered. Most importantly, it is entirely unclear how the MDR protein exhibits specificity for such a diverse range of substrates when, in bacteria, highly specific transport systems exist for each individual substrate to be transported. Related to this is the question of the identity of the physiological substrates and normal cellular roles of the MDR and CQR proteins. Drugs such as Adriamycin or chloroquine are clearly not normally encountered by the cells and for MDR, it is clear that simple amplification of the normal protein is sufficient to confer resistance 9 For CQR, it remains to be established whether simple amplification is responsible for resistance or whether the protein must be modified by mutation to enable it to handle chloroquine. The fact that CQR is stably inherited suggests that more than simple amplification may be involved.

In the elegant and intriguing paper in this issue ${ }^{2}, \mathrm{McGrath}$ and Varshavsky for the first time establish the normal cellular function of a eukaryotic member of the MDR protein family. However, rather than ending speculation, their unexpected findings serve to emphasize how little we actually know. They demonstrate that the STE6 gene of yeast, which is essential for the export of a-factor mating pheromone from the cell, encodes an MDR homologue. Although it remains a formal possibility that the inhibition of a-factor export is an indirect effect of STE6 mutations, the authors suggest that STE6 protein does, itself, mediate a-factor export by a process that differs from that used to secrete most yeast proteins. This raises many questions. Why should a-factor require a different export mechanism? Is $S T E 6$ used to export other proteins? And is STE6 the only protein required for the process to operate? Intriguingly, the HlyB protein of Escherichia coli required for haemolysin secretion shares considerable sequence similarity with both STE6 and the MDR protein, particularly in the ATP-binding domains, although the hydrophobic domains do differ somewhat, and, unlike the mammalian proteins, HlyB has just a single hydrophobic domain and a single ATP-binding domain ${ }^{5,6}$.

So, we have a rather unexpected paradox. MDR and many of its homologues in eukaryotes and prokaryotes appear to mediate the transport of small molecules across the membrane whereas other members of the family appear to mediate translocation of specific proteins. These two processes, while superficially the same, have generally been considered to involve entirely distinct mechanisms. This assumption may now need re-examination. Many surprises are likely to be in store.

Chris Higgins is in the Department of Biochemistry, University of Dundee, Dundee DD1 $4 H N, U K$.

1. Foote, S.J., Thompson, J.K., Cowman, A.F. \& Kemp, D.J. Cel/57, 921-930 (1989).

. McGrath, J.P. \& Varshavsky, A. Nature 340, 400-404 (1989).

3. Gros, P., Croop, J.M. \& Houseman, D. Cell 47, 371-380 (1986).

4. Chen, C. et al. Cell 47, 231-239 (1986)

5. Gerlach, J.H. et al. Nature 324, 485-489 (1986)

6. Higgins, C.F. et al. Nature 323, 448-451 (1986).

7. Higgins, C.F. et al. BioEssays 8, 111-116 (1988)

8. Arres, G. F-L. Cell 47, 323-324 (1986).

9. Gros, P., Neriah, Y.B., Croop, J.M. \& Houseman, D.E. Gature 323, 728-731 (1986) 